\title{
Thinking about Wahlpflicht: Anmerkungen zu einer überfälligen Diskussion*
}

\section{Diagnose: Rückläufige Wahlbeteiligung}

Der Befund ist eindeutig: Die Wahlbeteiligung in Deutschland geht zurück. Wie ein Blick auf Abbildung 1 zeigt, sind von dieser Entwicklung alle Ebenen des politischen Systems betroffen - Bundestags- und Landtags- ebenso wie Europawahlen. Lange Zeit schwankten die Wahlbeteiligungsraten in Deutschland in relativ festen Korridoren: Bei Bundestagswahlen gingen zwischen 80 und 90 Prozent der Menschen zur Wahl, bei Landtagswahlen zwischen 70 und 80 Prozent. Auch bei den ersten vier Europawahlen zwischen 1979 und 1994 bewegte sich die Wahlbeteiligung auf einem recht konstanten Niveau von rund 60 Prozent.

Seit den späten 1990er Jahren allerdings gelten diese Korridore nicht mehr: Die Wahlbeteiligung bei der Bundestagswahl 2009 lag nur noch knapp über 70 Prozent, bei Landtagswahlen werden häufig kaum mehr 60 Prozent erzielt. Zuweilen - so geschehen jüngst in Niedersachsen - wird schon ein geringer Anstieg der Wahlbeteiligung von 57,1 Prozent 2008 auf 59,4 Prozent 2013 als bemerkenswerter Erfolg gedeutet. Bei Europawahlen scheint selbst eine Marke von 50 Prozent derzeit kaum erreichbar.

Natürlich gibt es keinen objektiv wahren Blick auf solche Zahlen. Über die Frage, wie hoch die optimale Wahlbeteiligung ist, lässt sich trefflich streiten. Mit einem vergleichenden Blick etwa auf die Situation in den USA oder der Schweiz lässt sich argumentieren, dass altehrwürdige Demokratien gut funktionieren, auch wenn ihre Wahlbeteiligungsraten ein eher bescheidenes Niveau erreichen. Mit einem historischen Blick auf die Weimarer Republik lässt sich plausibel argumentieren, dass $\mathrm{(zu})$ hohe Wahlbeteiligungsraten auch dysfunktional und systemzersetzend sein können.

* Mein Dank gilt Cornelia Hentschel, Judith Schenk, Christian Schulze und Sven Vollnhals, die mich bei den Recherchen und der Endredaktion dieses Beitrags unterstützt haben, sowie Tobias Michael und Alexander Glantz von IPSOS für die mir eingeräumte Möglichkeit, Fragen zur Wahlpflicht im Ipsos Capibus zu stellen. 
Abbildung 1: Wahlbeteiligungsraten in Deutschland bei Bundestags-, Landtags- und Europawahlen

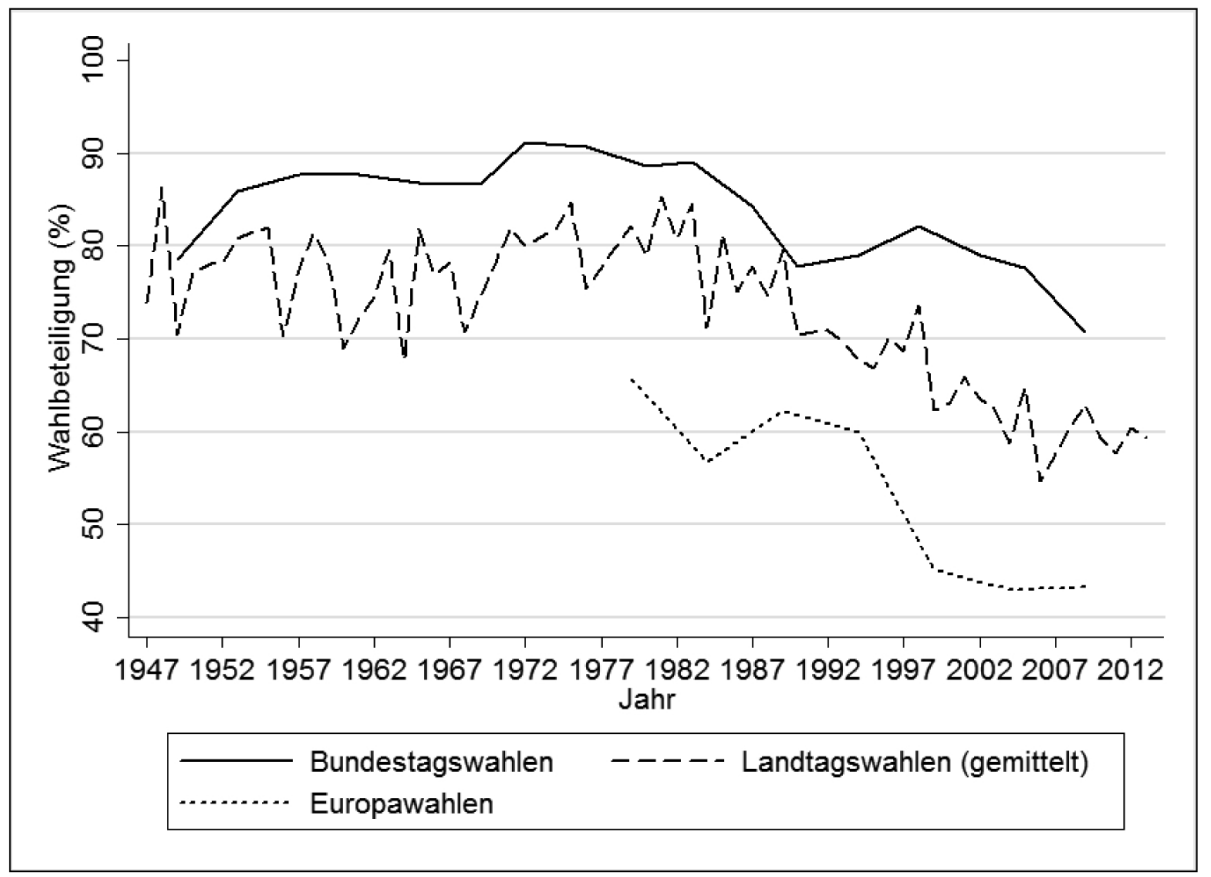

Anmerkung: In vielen Jahren hat mehr als eine Landtagswahl stattgefunden; für diese Jahre wurden die Werte gemittelt.

Dem stehen ebenso überzeugende normative Argumente gegenüber: In der Demokratie soll der demos das kratos haben, das Volk soll regieren - und nicht bloß Teile davon. In der Demokratie soll der Output des politischen Systems die Präferenzen der Bürgerinnen und Bürger reflektieren - und nicht bloß von ausgewählten Teilen. Oder um es mit den Worten amerikanischer Kollegen zu sagen: „Meaningful democratic participation requires that the voices of citizens in politics be clear, loud, and equal" (Verba/Schlozman/Brady 1995: 509). Denn nur gleich laute Stimmen sichern auch gleich starken Einfluss: „Who votes, and who doesn't, has important consequences for who gets elected and for the content of public policies" (Lijphart 1997: 4). Und selbst wenn es diese Konsequenzen nicht gäbe, so bliebe noch immer das alte Argument von John Stuart Mill, wonach politische Partizipation eben auch ein Wert an sich ist - ein Argument, dass sich auch in der derzeitigen Diskussion über „mehr Bürgerbeteiligung“ großer Beliebtheit erfreut. 
Thinking about Wahlpflicht: Anmerkungen zu einer überfälligen Diskussion

\section{Erklärungsmodelle zur Wahlbeteiligung}

Auch wenn sich über diesen Punkt trefflich streiten lässt, so sollte man gleichwohl nicht bloß phänotypisch auf die Wahlbeteiligung schauen, sondern auch - genotypisch - auf die zugrunde liegenden Ursachen. Die Gründe, die im Einzelfall zur Nichtwahl führen, können höchst unterschiedlicher Natur sein - sie haben aber Konsequenzen dafür, wie diese Nichtwahl zu bewerten ist. (Vor diesem Hintergrund ist übrigens auch die Metapher der „Partei der Nichtwähler“ jenseits eines einfachen Etiketts wertlos.) Es ist eben für die Bewertung des Phänomens sinkender Wahlbeteiligung nicht egal, ob eine wahlberechtigte Person aus freien Stücken (im Sinne der Freiheit der Wahl) darauf verzichtet, von ihrem Wahlrecht Gebrauch zu machen, oder ob strukturelle Gründe die Wahlbeteiligung verhindern.

Wirft man einen Blick in die einschlägige Literatur, so findet sich eine Reihe von Erklärungsfaktoren, die mit der Wahlbeteiligung ursächlich in Verbindung stehen. Für die hiesige Fragestellung eignet sich zur Systematisierung - gerade auch vor dem Hintergrund der normativen Argumente und ihrer Implikationen - die von Brady/Verba/Schlozman (1995: 271) vorgeschlagene Trias, wonach Wahlberechtigte von ihrem Wahlrecht keinen Gebrauch machen, ,because they can't, because they don't want to, or because nobody asked”. Der Aspekt des „Nicht-Könnens“ verweist dabei auf individuelle Eigenschaften, die als notwendige Bedingung für politische Partizipation gegeben sein müssen; Milbrath/Goel (1977) sprechen von facilitative factors. Dagegen verdeutlicht der Aspekt des „Nicht-Wollens“ die grundsätzliche Freiwilligkeit und damit die ebenfalls notwendige Motivation als weitere Voraussetzung politischer Partizipation; Milbrath/Goel (1977) sprechen von motivational factors. Dabei können die (de-)motivierenden Faktoren sehr unterschiedlicher Natur sein: Bin ich zufrieden und gehe deshalb nicht zur Wahl? Oder bin ich unzufrieden und gehe deshalb nicht zur Wahl (,weil ja eh alles nichts bringt"). Nobody asked schließlich weist darauf hin, dass es auch externe Einflüsse auf die individuelle Wahlbeteiligung gibt; darauf kommen wir sogleich zurück.

Vor allem die Unterscheidungen zwischen „Nicht-Können“ und „Nicht-Wollen“ hat wichtige normative Implikationen. Pointiert formuliert heißt es dazu bei Brady/ Verba/Schlozman (1995: 272): "If individuals eschew politics because they do not care - because they prefer to devote themselves to private rather than public pursuits - then we are apt to dismiss inactivity as a matter of personal choice. If the failure to get involved is the consequence of resource constraints that make it difficult for even those who are politically interested and engaged to take part in political life, then we are likely to be more concerned about political inactivity". Und auch ein bewusster Verzicht auf die Wahlteilnahme, ,weil ja eh alles nichts bringt“, wäre 
gänzlich anders zu bewerten als ein freiwilliger Verzicht aus der Motivation heraus, dass alles gut laufe und kein korrigierender Eingriff nötig sei.

All dies wirft die Frage nach der Empirie auf. Was zeigen (jüngere) Forschungsergebnisse zu den Hintergründen der Wahlbeteiligung? Hinweise, dass Nichtwahl etwas mit Zufriedenheit oder Normalisierung zu tun habe, sind dabei rar. Dagegen findet sich eine Reihe von Beiträgen, die die Wahlbeteiligung in engem Zusammenhang mit Unzufriedenheit (mit dem politischen Personal, aber auch dem politischen System insgesamt) sehen. Und es gibt starke Hinweise auf strukturelle Hindernisse. Immer wieder ist gezeigt worden, dass Nichtwahl mit niedrigerer formaler Bildung, aber auch mit einer prekären wirtschaftlichen Lage verbunden ist. Dies gilt mit Blick auf Individuen (Faas 2010); in jüngerer Vergangenheit hat aber insbesondere auch Armin Schäfer eindrucksvoll nachweisen können, wie eng der Zusammenhang zwischen der wirtschaftlichen Situation und der Wahlbeteiligung auf der Ebene von Stadtteilen ist: Je angespannter die wirtschaftliche Lage ist, desto niedriger ist in den Stadtteilen auch die Wahlbeteiligung (Schäfer 2012, Faas 2012). Die von Lipset (1960: 192) früh formulierte Erwartung, wonach ,[g]roups subject to economic pressures with which individuals cannot cope [...] might also be expected to turn to government action as a solution", trifft also gerade nicht $\mathrm{zu}$. Alles in allem kann man jedenfalls im Lichte empirischer Forschung die niedrige Wahlbeteiligung kaum mit einem Schulterzucken und dem Verweis auf Zufriedenheit und/oder die Freiheit der (Nicht-)Wahl abtun.

Erfreulicherweise nennen Brady/Verba/Schlozman (1995) aber nicht bloß die Faktoren des „Nicht-Könnens“ und „Nicht-Wollens“, sondern verweisen auch auf den Aspekt des nobody asked. Anders formuliert: Sie betrachten Wahlbeteiligung nicht bloß als ein von der Nachfrageseite getriebenes Phänomen, sondern berücksichtigen auch explizit die politische Angebotsseite, denn natürlich können auch Parteien und andere Akteure der Mesoebene (in aller Regel) mobilisierend wirken und damit eventuell vorhandene motivationale oder strukturelle Defizite der Bürgerinnen und Bürger ausgleichen - wenn sie dies denn möchten.

Dass der Kontext mit Blick auf die Wahlbeteiligung eine wichtige Rolle spielt, wissen wir auch aus der einschlägigen international vergleichenden Forschung. Einige wenige Systemvariablen - allen voran konkrete Ausprägungen des Parteienund Wahlsystems - genügen in der Regel, um die beachtlichen Unterschiede, die sich zwischen verschiedenen Ländern hinsichtlich der beobachtbaren Wahlbeteiligungsraten ergeben, gut erklären zu können. Dass solche Kontexteffekte zu beobachten sind, weist erneut daraufhin, dass individuelle Faktoren (wie das Nicht-Können und das Nicht-Wollen) mit dem jeweiligen (institutionellen) Kontext interagieren, genau wie es die fundamental equation of politics (Hinich/Munger 1997) auch 
erwarten ließe: Beobachtbare Politikergebnisse sind demnach das Ergebnis der Interaktion individueller Präferenzen und dem jeweiligen institutionellen Kontext.

Das gilt gerade auch im Kontext der Wahlbeteiligung. Der institutionelle Kontext hat einen prägenden Einfluss auf die durchschnittliche Höhe der Wahlbeteiligung in einzelnen Ländern - mit Folgen für Individualfaktoren, denn ,the effects of individual characteristics do depend on how much space is left for such characteristics to play a role after institutional and political contexts have defined the overall level of participation" (Franklin/van der Eijk/Oppenhuis 1996: 321). Darüber hinaus konnten empirische Analysen auch genau den skizzierten Interaktionseffekt zwischen den Kontextfaktoren und den individuellen Kalkülen der Wähler (siehe Perea 2002) nachweisen. Gabriel/Völkl (2004) sprechen von der „Gelegenheitsstruktur der Wahlbeteiligung“, die durch die Institutionen geschaffen wird.

Wenn wir im Kontext von Wahlbeteiligung von potenziellen Folgen des institutionellen Rahmens für individuelles Handeln sprechen, so sollte man an dieser Stelle die Überlegungen keineswegs nur auf die Wahlberechtigten beschränken. Auch die „politischen Unternehmer“ - Kandidaten, Funktionäre, Wahlkampfmanager - werden all dies in ihren (strategischen) Überlegungen berücksichtigen. Das zeigt übrigens auch das Modell der second-order-elections von Reif/Schmitt (1980) sehr schön, das eben nicht bloß über die Wähler spricht, sondern auch Medien, Parteien und Politiker explizit berücksichtigt. Auch im Sinne des nobody asked passiert in diesen Nebenwahlen eben weniger.

\section{Wahlpflicht als Institution des Wahlsystems}

Die Analysen zu den institutionellen Einflussfaktoren auf die Wahlbeteiligung fördern regelmäßig einen geradezu dominanten Faktor zutage: die Wahlpflicht. In Ländern mit Wahlpflicht liegt die Wahlbeteiligung erheblich höher als in Ländern ohne Wahlpflicht - auch wenn sie selbst dort die 100 Prozent nicht erreicht.

Das World Factbook der CIA (2013) listet 23 Länder auf, in denen eine Wahlpflicht existiert: Ägypten, Argentinien, Australien, Belgien, Bolivien, Brasilien, Chile (dort ist sie allerdings 2011 abgeschafft worden), Costa Rica, Demokratische Republik Kongo, Dominikanische Republik, Ecuador, Griechenland, Honduras, Libanon, Luxemburg, Mexiko, Nauru, Panama, Paraguay, Peru, Singapur, Thailand und Uruguay. Dabei gilt in einigen (vor allem südamerikanischen) Ländern die Pflicht allerdings nur bis zu einem bestimmten Höchstalter, in der Regel von 70 Jahren. Zu ergänzen sind zu dieser Liste noch Italien, das in Artikel 48 noch immer von „Wählen als Bürgerpflicht" spricht, sowie Zypern (siehe auch www.cses.org und Robbe 2009). Auch Österreich und die Niederlande haben historische Erfah- 
rungen mit der Wahlpflicht; im Schweizer Kanton Schaffhausen gilt sie ebenfalls bis heute. Und Artikel 26 (3) der Landesverfassung von Baden-Württemberg lautet: „Die Ausübung des Wahl- und Stimmrechts ist Bürgerpflicht.“ Schaut man sich die Liste der Länder an, so kann man eigentlich nur feststellen: Es ist ein bunter Strauß (mit leicht südamerikanischem Einschlag). ${ }^{1}$

Gleichwohl zeigen die international vergleichenden Studien, dass die Existenz einer Wahlpflicht Folgen hat. Die Menschen reagieren offenkundig auf diese institutionellen Anreize (und gewöhnen sich daran). In der Folge liegt die Wahlbeteiligung höher; außerdem sind die Strukturen der Wahlbeteiligung deutlich weniger sozial selektiv - allerdings wird dafür staatlicher Zwang eingesetzt.

Und es würden eben nicht bloß die Wahlberechtigten darauf reagieren. Im Sinne des nobody asked würde sich auch die Anreizstruktur für politische Eliten grundlegend verändern, wenn es eine Wahlpflicht gäbe. ${ }^{2}$ Strategien einer ,asymmetrischen Demobilisierung" würden per definitionem nicht mehr greifen. Im Gegenteil: Weil davon auszugehen wäre, dass sich die Zahl der Wählerinnen und Wähler deutlich erhöhen würde, müssten sich Parteien und Politiker auch wieder um all jene kümmern und sorgen, um die sie sich heute eher am Rande bemühen. Zugespitzt formuliert könnte man erwarten: Mit einer Wahlpflicht würde sich die Distanz zwischen Wählern und Gewählten reduzieren, weil beide sich bewegen müssten.

\section{Ist das realistisch?}

Natürlich bleibt die Frage, wie realistisch eine Wahlpflicht in Deutschland ist. Eine Wahlpflicht schon seit langer Zeit zu haben (wie es in den zuvor genannten Ländern der Fall ist), mag das eine sein. Eine Wahlpflicht neu einzuführen, ist das andere.

Zunächst einmal ist festzuhalten, dass es aus dem Kreise der Politikwissenschaft immer wieder Forderungen nach Einführung einer Wahlpflicht gibt. Arend Lijphart etwa hat in seiner presidential address als Präsident der American Political Science Association - in Analogie zum preußischen Drei-Klassen-Wahlrecht, welches heutzutage universell als undemokratisch abgelehnt würde - gefragt: ,Why then do ma-

1 Vielfalt gibt es übrigens auch mit Blick auf mögliche Sanktionierungen, die im Falle eines Verstoßes gegen die Wahlpflicht greifen. In den Daten der „Comparative Study of Electoral Systems“ etwa werden drei Stufen - strikter Vollzug der Sanktionen, laxer Vollzug der Sanktionen, kein Vollzug von Sanktionen - unterschieden.

2 Mit Theodor Heuss könnte man natürlich auch Sorge vor einer „Prämie für jeden Demagogen“ haben, der die Frustrierten und Unzufriedenen, die per Wahlpflicht zur Stimmabgabe gezwungen werden, mit seinen Parolen für sich gewinnen könnte. In einem outputorientierten Sinne ließe sich zweifelsohne so argumentieren. Inputorientiert aber ist das Abschieben von Teilen des Elektorats in die Nichtwahl nicht minder bedenklich. 
ny democrats tolerate the systematic pattern of low and unequal turnout that is the functional equivalent of such rules?" Die Lösung liegt für ihn in der Einführung einer Wahlpflicht. Zu ähnlichen Schlussfolgerungen kommen auch Engelen (2007) und Keaney/Rogers (2006). ${ }^{3}$ Auch in Deutschland gibt es Forderungen, über die Einführung einer Wahlpflicht zu diskutieren: Merkel/Petring (2012: 116) etwa konstatieren. „Mit der Wahlpflicht existiert ein Mechanismus, der die Wahlbeteiligung massiv anhebt und die soziale Verzerrung stark reduziert.“ Auch Armin Schäfer kommt auf der Basis seiner Analysen zur Wahlbeteiligung zum Schluss, dass man ernsthaft über eine Wahlpflicht nachdenken solle (Schäfer 2009).

Dem schließe ich mich voll an: Eine Diskussion über Wahlpflicht ist überfällig. Daran ändert auch die Tatsache nichts, dass entsprechende Vorstöße aus dem Kreise von Politikern gerne als „Sommerloch“-Themen abgetan werden. Spätestens mit der verfassungsrechtlichen Keule, wonach eine Wahlpflicht nicht verfassungskonform wäre, da sie die Freiheit der Wahl einschränken würde, ist das Thema zumeist erledigt. Zuletzt ist es dem FDP-Politiker Heinrich Heidel - seines Zeichens immerhin der Vizepräsident des hessischen Landtags - so ergangen, 2009 machten der CSU-Abgeordnete Stephan Mayer und der SPD-Abgeordnete Jörn Thießen ähnliche Erfahrungen.

Es handelt sich um einen Zielkonflikt, wie auch Merkel/Petring (2012: 116) betonen. Ihrer Meinung nach würde Wahlpflicht bedeuten: „Zugunsten der demokratischen Gleichheit findet ein Eingriff in die individuelle Wahlfreiheit statt. Auch wenn dieser Eingriff sicherlich minimal ist, ist die Problematik nicht völlig von der Hand zu weisen. [...] Der demokratietheoretische Gütertausch heißt: minimale Freiheitseinschränkung gegen beachtliche politische Gleichheitsgewinne." Auch in einer Publikation des wissenschaftlichen Dienstes des Deutschen Bundestages ist zu lesen, dass , die verfassungsrechtliche Zulässigkeit einer Wahlpflicht [...] streitig diskutiert [wird]“. Weiter heißt es dort: „Unstreitig ist, dass eine Wahlpflicht durch eine Verfassungsänderung eingeführt werden könnte“ (Robbe 2009).

Letztlich wäre es eine politische Entscheidung, die selbst auf demokratischem Wege gefunden werden müsste. Das setzt zweierlei voraus: Unterstützung auf Seiten der politischen Parteien und Unterstützung in der Bevölkerung, die von dieser Maßnahme natürlich zuvorderst betroffen wäre.

3 Es gibt natürlich auch kritische Stimmen, die vor allem darauf abzielen, dass sich letztlich an den Ergebnissen der Politik gar nicht so viel ändern würde (da sich die Präferenzen von Wählern und Nichtwählern eben gar nicht so stark unterscheiden) und die daher die Option des Zwangs nicht ziehen möchten (Lutz/Marsh 2007 mit den weiteren Artikel des Special Issues der Electoral Studies; siehe auch Bennett/Resnick 1990). 
Was die Parteien betrifft, gibt es zunächst wenig Hinweise auf Unterstützung einer Wahlpflicht, sieht man einmal von punktuellen Forderungen von Einzelpersonen ab. Systematische Evidenz liegt allerdings kaum vor, daher lohnt ein Blick in die Kandidatenstudie 2002. ${ }^{4}$ Dort nämlich wurden die bei dieser Bundestagswahl antretenden Direktkandidaten von Union, SPD, FDP, Grünen und (damals) PDS zu ihrer Haltung zu der Aussage: ,Es sollte die Wahlpflicht eingeführt werden“ gefragt. 729 Direktkandidaten haben diese Frage beantwortet; insgesamt 68 Prozent lehnen sie ,eher“ oder „vollkommen“ ab. Dem stehen nur 23 Prozent gegenüber, die der Aussage „voll und ganz" oder ,eher“ zustimmen. Dabei halten sich auch die Unterschiede zwischen Parteien in engen Grenzen: Am wenigsten stark lehnen die Kandidaten der SPD die Wahlpflicht ab (58 Prozent Ablehnung), am vehementesten tun es die Kandidaten der FDP (79 Prozent Ablehnung). Insgesamt also war von Seiten der Parteien - zumindest 2002 - kaum Unterstützung für die Idee einer Wahlpflicht zu erwarten.

Auch was die Bevölkerung selbst betrifft, liegen Umfrageergebnisse vor, an dieser Stelle sogar aus jüngster Vergangenheit. Jüngst sind im Rahmen einer repräsentativen Studie zufällig ausgewählten Befragten folgende drei Aussagen präsentiert worden: ${ }^{5}$

- In Deutschland sollte die Wahlpflicht eingeführt werden.

- Wer an einer Wahl nicht teilnimmt, sollte ein Bußgeld zahlen müssen.

- Wer am Wahltag seine Stimme abgibt, sollte dafür eine kleine Belohnung erhalten.

Die Antworten sollten auf einer fünfstufigen Skala von ,stimme überhaupt nicht zu“ (-2) bis ,stimme voll und ganz zu“ (+2) gegeben werden. Abbildung 2 zeigt die entsprechenden Ergebnisse. Auch hier dominiert insgesamt die Skepsis gegenüber einer Wahlpflicht - und zwar nicht nur als solcher, sondern auch in Form möglicher Implementationen, nämlich negativer Sanktionen (Bußgeld) als auch positiver Anreize (finanzielle Belohnung). Gerade die Vorstellung, man müsse bei Nichtwahl ein Bußgeld bezahlen, wird strikt abgelehnt und findet praktisch keinerlei Zustimmung.

4 Der entsprechende Datensatz kann über das GESIS Datenarchiv unter der Nummer 4225 bezogen werden.

5 Die Fragen sind im Rahmen des monatlichen Ipsos Capibus gestellt worden. Die Feldzeit dieser Befragung war vom 7. bis 13. Januar 2013; insgesamt wurden 953 zufällig ausgewählte Personen persönlich (CAPI) befragt. 


\section{Abbildung 2: Einstellungen zur Wahlpflicht in Deutschland}

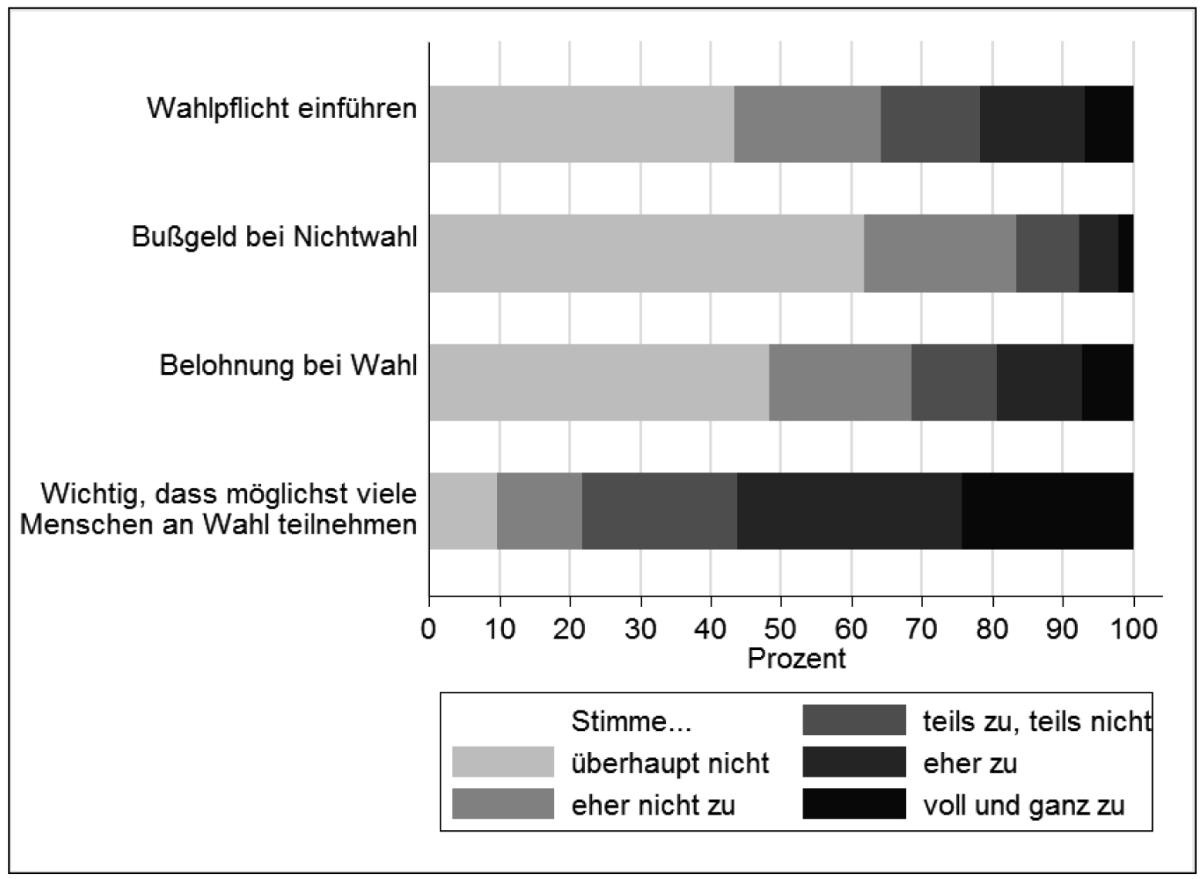

Quelle: Ipsos Capibus (Januar 2013)

Die Diskussion damit allerdings für beendet zu erklären, ehe sie überhaupt angefangen hat, wäre verfrüht. Denn in der gleichen Umfrage ist zudem nach der Zustimmung bzw. Ablehnung der Aussage „Es ist mir wichtig, dass möglichst viele Menschen an einer Wahl teilnehmen" gefragt worden. Auch die Antwortvorteilung dazu findet sich in Abbildung 2 - und hier überwiegt klar die Zustimmung. Es ist also keineswegs so, dass die Menschen im Land nicht sensibel für die (Höhe der) Wahlbeteiligung wären. Einzig eine Wahlpflicht scheint derzeit weder aus Sicht der Parteien noch der Mehrheit der Bevölkerung ein geeignetes Instrument dafür zu sein. 


\section{Ausblick}

Dass in Deutschland eine Wahlpflicht eingeführt wird, ist vor dem Hintergrund dieser empirischen Befunde derzeit wenig wahrscheinlich. Eine Diskussion über Wahlpflicht macht dies allerdings keinesfalls entbehrlich. Sie würde ihr endlich einen angemessenen Platz im Diskurs schaffen und den Finger in die Wunde der niedrigen Wahlbeteiligung legen, deren oben skizzierte Hintergründe auch ungeachtet der präsentierten Umfrageergebnisse problematisch bleiben.

Schließlich bleibt die Frage: Muss es zwangsläufig Zwang sein? Die Einführung einer Pflicht ist immer nur ein extreme Option, darunter liegt eine ganze Dimension von Möglichkeiten, die man in Angriff nehmen könnte, um den Wahlakt wieder attraktiver zu machen und damit die Distanz zwischen Wählern und Gewählten zu verkleinern.

Dass man sich über viele Monate und Jahre mit Details des Wahlrechts beschäftigen kann, hat nicht zuletzt die Diskussion um das negative Stimmengewicht und die Überhangmandate gezeigt. Leider hat man dabei die Chance verpasst, auch über andere Reformmöglichkeiten des Wahlrechts - im Sinne einer Attraktivitätssteigerung - nachzudenken. Da die Erwartung allerdings allseits ist, dass das neue Wahlrecht nur ein einziges Mal zur Anwendung kommen wird, um anschließend durch ein neues Wahlrecht ersetzt zu werden, bietet sich dann die Chance erneut. Würde man ähnlich intensiv über funktionale Äquivalente einer Wahlpflicht nachdenken, wie man zuvor über Lösungen des negativen Stimmengewichts nachgedacht hat es gäbe sicherlich viele gute Ideen. Die Wahlbeteiligung jedenfalls muss raus aus ihrem Mauerblümchendasein. Das ist den Bürgerinnen und Bürgern - auch das haben die Umfrageergebnisse gezeigt - wichtig. Und dagegen darf auch keine Partei sein. Eine Diskussion darüber ist überfällig. Ideen werden gebraucht. Die Grünen übrigens haben sich gerade für die Bundestagswahl 2013 ein Wahlziel von sechs Millionen Stimmen gesetzt. Eine charmante Idee. Absolutzahlen an Stimmen sind nämlich auch ein Weg, um den Finger in die Wunde der sinkenden Wahlbeteiligung zu legen. 
Thinking about Wahlpflicht: Anmerkungen zu einer überfälligen Diskussion

\section{Literatur}

Bennett, Stephen Earl/Resnick, David, 1990: The Implications of Nonvoting for Democracy in the United States, in: American Journal of Political Science 34, 771-802.

Brady, Henry E./Verba, Sidney/Schlozman, Kay Lehman, 1995: Beyond SES: A Resource Model of Political Participation, in: American Political Science Review 89, 271-294.

CIA, 2013: The World Factbook, Washington, https://www.cia.gov/library/publica tions/the-world-factbook/index.html (12.2.2013).

Engelen, Bart, 2007: Why Compulsory Voting Can Enhance Democracy, in: Acta Politica 42, 23-39.

Faas, Thorsten, 2010: Arbeitslosigkeit und Wählerverhalten, Baden-Baden.

Faas, Thorsten, 2012: Wahlbeteiligung, in: Jan W. van Deth/Markus Tausendpfund (Hrsg.), Politik im Kontext: Individuelle und kontextuelle Determinanten politischer Orientierungen, Wiesbaden, 413-440.

Franklin, Mark N./van der Eijk, Cees/Oppenhuis, Erik, 1996: The Institutional Context: Turnout, in: Cees van der Eijk/Mark Franklin (Hrsg.), Choosing Europe? The European Electorate and National Politics in the Face of the Union, Ann Arbor, 306-331.

Gabriel, Oscar W./Völkl, Kerstin, 2004: Auf der Suche nach dem Nichtwähler neuen Typs: Eine Analyse aus Anlass der Bundestagswahl 2002, in: Frank Brettschneider/Jan van Deth/Edeltraud Roller (Hrsg.), Die Bundestagswahl 2002: Analysen der Wahlergebnisse und des Wahlkampfs, Wiesbaden, 221-248.

Hinich, Melvin J./Munger, Michael C., 1997: Analytical Politics, Cambridge.

Keaney, Emily/Rogers, Ben, 2006: A Citizen's Duty: Voter Inequality and the Case for Compulsory Turnout, London.

Lijphart, Arend, 1997: Unequal Participation: Democracy's Unresolved Dilemma, in: American Political Science Review 91, 1-14.

Lipset, Seymour Martin, 1960: Political Man, Garden City.

Lutz, Georg/Marsh, Michael, 2007: Introduction: Consequences of Low Turnout, in: Electoral Studies 26, 392-400.

Merkel, Wolfgang/Petring, Alexander, 2011: Partizipation und Inklusion, Berlin, http://www.demokratie-deutschland-2011.de/partizipation-und-inklusion.php (13.2.2013).

Merkel, Wolfgang/Petring, Alexander, 2012: Politische Partizipation und demokratische Inklusion, in: Tobias Moerschel/Christian Krell (Hrsg.), Demokratie in Deutschland, Wiesbaden, 93-119. 
Milbrath, Lester W./Goel, M. L., 1977: Political Participation: How and Why Do People Get Involved in Politics?, Lanham.

Perea, Eva Anduiza, 2002: Individual Characteristics, Institutional Incentives and Electoral Abstentions in Western Europe, in: European Journal of Political Research 41, 643-673.

Reif, Karlheinz/Schmitt, Hermann, 1980: Nine Second-Order National Election - a Conceptual Framework for the Analysis of European Election Results, in: European Journal of Political Research 8, 3-44.

Robbe, Patrizia, 2009: Wahlpflicht. Veröffentlichung des Wissenschaftlichen Dienstes des Deutschen Bundestags 61/2009, Berlin.

Schäfer, Armin, 2009: Alles halb so schlimm? Warum eine sinkende Wahlbeteiligung der Demokratie schadet, in: MPIfG Jahrbuch 2009/2010, Köln, 33-38.

Schäfer, Armin, 2012: Beeinflusst die sinkende Wahlbeteiligung das Wahlergebnis?

Eine Analyse kleinräumiger Wahldaten in deutschen Großstädten, in: Politische Vierteljahresschrift 53, 240-264.

Verba, Sidney/Schlozman, Kay Lehman/Brady, Henry E., 1995: Voice and Equality:

Civic Voluntarism in American Politics, Cambridge.

Korrespondenzanschrift:

Prof. Dr. Thorsten Faas

Johannes Gutenberg-Universität Mainz

Institut für Politikwissenschaft

Bereich "Methoden der empirischen Politikforschung"

Hegelstr. 59

55122 Mainz

E-Mail: thorsten.faas@googlemail.com 